Canadian

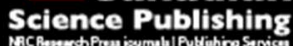

Applied Physiology, Nutrition, and Metabolism Physiologie appliquée, nutrition et métabolisme

\title{
Moderate Physical Activity Promotes Basal Hepatic Autophagy in Diet-Induced Obese Mice
}

\begin{tabular}{|c|c|}
\hline Journal: & Applied Physiology, Nutrition, and Metabolism \\
\hline Manuscript ID & apnm-2016-0280.R2 \\
\hline Manuscript Type: & Article \\
\hline Date Submitted by the Author: & 15-Aug-2016 \\
\hline Complete List of Authors: & $\begin{array}{l}\text { Rosa-Caldwell, Megan; University of Arkansas Fayetteville, Health, Human } \\
\text { Performance and Recreation; } \\
\text { Lee, David; University of Arkansas Fayetteville, Health, Human } \\
\text { Performance and Recreation } \\
\text { Brown, Jacob; University of Arkansas Fayetteville, Health, Human } \\
\text { Performance and Recreation } \\
\text { Brown, Lemuel; University of Arkansas, Health, Human Performance, and } \\
\text { Recreation } \\
\text { Perry, Jr, Richard; University of Arkansas Fayetteville, Health, Human } \\
\text { Performance and Recreation } \\
\text { Greene, Elizabeth; University of Arkansas Fayetteville, Center of Excellence } \\
\text { for Poultry Science } \\
\text { Carvallo Chaigneau, Francisco; University of California System } \\
\text { Washington, Tyrone; University of Arkansas, Health, Human Performance, } \\
\text { and Recreation } \\
\text { Greene, Nicholas; University of Arkansas Fayetteville, Human Health } \\
\text { Performance and Recreation }\end{array}$ \\
\hline Keyword: & $\begin{array}{l}\text { diabetes, exercise, non-alcoholic fatty liver disease, insulin resistance, } \\
\text { mitochondria }\end{array}$ \\
\hline
\end{tabular}

\section{SCHOLARONE" \\ Manuscripts}




\section{Moderate Physical Activity Promotes Basal Hepatic Autophagy in Diet-Induced Obese}

Mice

Megan E. Rosa-Caldwell ${ }^{1}$, David E. Lee ${ }^{1}$, Jacob L. Brown ${ }^{1}$, Lemuel A. Brown ${ }^{2}$, Richard A.

Perry $\mathrm{Jr}^{2}$, Elizabeth S. Greene ${ }^{3}$, Francisco R Carvallo Chaigneau ${ }^{4}$, Tyrone A. Washington ${ }^{2}$,

Nicholas P. Greene ${ }^{1}$

${ }^{1}$ Integrative Muscle Metabolism Laboratory, Human Performance Laboratory, Department of

Health, Human Performance and Recreation, University of Arkansas, Fayetteville, AR 72701,

United States of America,mrosa@uark.edu, davidlee@uark.edu,jb024@uark.edu

${ }^{2}$ Exercise Muscle Biology Laboratory, Human Performance Laboratory, Department of Health,

Human Performance and Recreation, University of Arkansas, Fayetteville, AR 72701, United

States of America, 1xb028@uark.edu,rap0072@uark.edu,tawashin@uark.edu

${ }^{3}$ Center of Excellence for Poultry Science, University of Arkansas, Fayetteville, AR 72701,

United States of America, esgreene@uark.edu

${ }^{4}$ California Animal Health and Food Safety Laboratory, University of California-Davis School of Veterinary Medicine, Davis, CA 95616, United States of America, fcarvallo@ucdavis.edu

Corresponding Author: Nicholas P. Greene

University of Arkansas

155 Stadium Drive

321 HPER

Fayetteville, Arkansas 72701

Email: npgreene@uark.edu

Phone: 479-575-6638

Fax: 479-575-2853 


\begin{abstract}
:
Obesity is a known risk factor for the development of hepatic disease; obesity-induced fatty liver can lead to inflammation, steatosis and cirrhosis and is associated with degeneration of the mitochondria. Lifestyle interventions such as physical activity may ameliorate this condition.

Purpose: The purpose of this study was to investigate regulation of mitochondrial and autophagy quality control in liver following Western Diet-induced obesity and voluntary physical activity. Methods: 8 wk old C57BL/6J mice were fed Western Diet (WD) or normal chow (NC, control) for 4 weeks; afterwards, groups were divided into voluntary wheel running (VWR) or sedentary (SED) conditions for an additional 4 weeks. Results: WD-SED animals had a median histology score of 2, whereas WD-VWR was not different from NC groups (median score 1). There was no difference in mRNA of inflammatory markers $I l 6$ and Tnf $\alpha$ from WD. WD animals had 50\% lower mitochondrial content (COX-IV and Cytochrome C protein), 50\% lower Pgcl $\alpha$ mRNA content as well as reduced content of mitochondrial fusion and fission markers. Markers of autophagy were increased in VWR animals, regardless of obesity, as measured by $50 \%$ greater LC3II/I ratio and $40 \%$ lower p62 protein content. BNIP3 protein content was $30 \%$ less in WD animals compared to NC animals regardless of physical activity. Conclusions: Diet-induced obesity results in derangements in mitochondrial quality control which appear to occur prior to the onset of hepatic inflammation. Moderate physical activity appears to enhance basal autophagy in the liver, this increased autophagy may provide protection from hepatic fat accumulation.
\end{abstract}

Keywords: diabetes, exercise, Non-alcoholic Fatty Liver Disease, insulin resistance, mitochondria 


\section{Introduction}

The United States is chronically underactive and overfed, specifically in the consumption of fats and sugars, leading to a dramatic increase in obesity and Type 2 Diabetes Mellitus (T2DM) (Centers for Disease Control 2007). Obesity is a risk factor for the development of fatty liver disease, the diagnosis of which has correspondingly risen with obesity (Almeda-Valdes et al. 2014). Liver derangements as a result of obesity often include: reduced mitochondrial $\beta$ oxidation, oxidative stress, inflammation and reduced cellular health (Lavoie et al. 2006a). Hepatic lipid accumulation can begin as nonalcoholic fatty liver disease (NAFLD), but progress to nonalcoholic steatohepatitis (NASH), cirrhosis and eventually liver failure (Mokdad AH 2003). Currently, there are no pharmacological treatments once NASH has developed. Therefore, it is necessary to better define the hepatic derangements that occur in pre-steatotic conditions.

Mitochondria appear to be one of the key regulators of hepatocyte health. Insulin sensitivity is correlated with mitochondrial health in muscle and liver (Pessayre 2007); therefore, maintenance of mitochondrial quality, which we define as the health and functional ability of the mitochondrial network, may be imperative to preserve cellular function. Mitochondrial quality is regulated by multiple processes including mitochondrial biogenesis, dynamics, and mitochondrial autophagy (mitophagy) - mitochondrial quality control. Prior research suggests aberrant mitochondrial biogenesis following high-fat or high-sugar diets in animal models in liver (Rector et al. 2010; Rector et al. 2011). Mitochondrial dynamics refer to the fusion of mitochondria to share cellular components and the removal of damaged mitochondrial components through the process of fission (Westermann 2010). Previous work investigating mitochondrial dynamics in liver models with relation to high fat feeding have found detrimental 
increases in mitochondrial fission (Galloway et al. 2014; Xu et al. 2014; Wang et al. 2015). Yet, the impacts of exercise and physical activity (PA) on hepatic mitochondrial dynamics warrants further investigation.

Finally, selective autophagy, a primary mechanism for removal of damaged proteins and organelles (Mizushima et al. 2008), is key to maintenance of cellular function. Various studies have suggested that autophagy may be an important regulatory mechanism for the progression of obesity-induced NAFLD (Lavallard and Gual 2014). Glick et. al (Glick et al. 2012) demonstrated that knockout of BNIP3, a gene imperative for mitophagy, in young mice resulted in liver steatosis compared to their wild type littermates under normal feeding conditions, indicating the importance of mitophagy in hepatic health. However, the impacts of lipid-induced toxicity on hepatic autophagy have not been fully elucidated, as both increases and decreases in autophagy have been noted following lipid overload (Cai et al. 2014; Baena et al. 2015). Lira et al. (2013) have demonstrated in muscle that exercise training enhances basal autophagy, including mitophagy, which appears necessary for training-induced adaptations. Recently, Gonclaves et al. (2016) have found voluntary wheel running and treadmill running to improve derangements in hepatic autophagy during NASH conditions. However, the role of PA with regard to hepatic autophagy function warrants further investigation during pre-steatosis conditions.

We recently examined alterations in mitochondrial quality control in murine skeletal muscle following Western Diet (WD)-induced obesity and moderate voluntary wheel running (VWR) (Greene et al. 2015). However, in the liver, these research avenues have not yet been pursued. As impaired mitochondrial quality appears to be a major contributor to reductions in hepatocyte health, understanding the etiology of mitochondrial degeneration under conditions conducive to developing NAFLD may hold the key in developing new and efficacious 
therapeutic strategies for this disease. As such, we sought to define potential mitochondrial regulatory defects in the liver following induction of obesity and whether moderate lifestyle PA may ameliorate these derangements.

\section{Materials and Methods}

\section{Animals and interventions}

All protocols were approved by the University of Arkansas Institutional Animal Care and Use Committee. We have previously reported phenotypic data on these animals including body weight, fat weight, exercise and glucose tolerance, as well as muscle mitochondrial quality control (Greene et al. 2015). Wild-type C57BL/6J mice were purchased at seven weeks of age (Jackson Laboratories, Bar Harbor, ME) and housed in a secure, temperature and humidity controlled environment on a 12:12 hour light-dark cycle with chow and water provided $a d$ libitum. To induce obesity, at eight weeks of age, animals were divided into two groups; normal chow (NC, 54\% CHO, 17\% fat, 29\% protein, Teklad, Indianapolis, IN, Product \#8640) and Western diet-induced obesity (WD, $42 \%$ fat, $42.7 \% \mathrm{CHO}, 15.2 \%$ protein, $1.5 \mathrm{~g} / \mathrm{kg}$ of cholesterol, Research Diets Inc., New Brunswick, NJ, Product \# D12079B) similar to previous studies (Funai et al. 2013). After four weeks of diet, both groups were further subdivided into voluntary wheel running (VWR) or sedentary (SED) groups. This design resulted in four final experimental groups: NC-SED, NC-VWR, WD-SED and WD-VWR, n=10 per group. Each animal was singly housed and VWR animals were provided a free moving running wheel. Usage of the running wheel was monitored with Hall Effect Sensors connected to an 8-channel wheel counter interface with appropriate software (Columbus Instruments, Columbus, $\mathrm{OH}$ ). For the WD-VWR group two animals were identified who did not perform wheel running, therefore, data from these two 
animals were excluded for all subsequent analyses. To control for the effects of acute exercise, running wheels were removed 24 hours before euthanasia. Animals were euthanized at 16 weeks of age using $\mathrm{CO}_{2}$ asphyxiation and with confirmation by cervical dislocation. Tissues were quickly collected and snap-frozen in liquid nitrogen for later analysis. Mouse tibias were concurrently collected and assessed for total length as an estimate of total body size free of WDinduced weight gain (Perfield et al. 2013) and used previously in our laboratory (Greene et al. 2015); tibia length did not differ among groups.

RNA isolation, cDNA synthesis and real time RT-PCR

Livers were homogenized as previously described (Greene NP 2014). Briefly, samples were homogenized in Trizol reagent (Life Technologies, Grand Island, NY) and isolated using a commercially available kit (Life Technologies Ambion PureLink RNA Mini Kit, Grand Island, NY). RNA was then DNase treated, and purity and concentrations were determined using 260/280nm ratios read on a BioTek Take3 microvolume microplate with a BioTek PowerWave XC microplate reader (BioTek Instruments Inc., Winooski, VT). Isolated RNA was reverse transcribed into cDNA using $1 \mu \mathrm{g}$ of RNA and Quanta qScript cDNA Supermix (Quanta BioSceinces, Gaithersburg, MD) according to manufacturer's instructions. cDNA was diluted to $10 \mathrm{ng} / \mu \mathrm{L}$, and gene expression was analyzed using a Step-One real-time RT-PCR instrument (Applied BioSystems) and data was analyzed using StepOne Software. For real-time RT-PCR, cDNA was amplified in a reaction containing appropriate primer pairs or probes and SYBR Green or TaqMan Universal Master mix as appropriate (Greene et al. 2015). PCR reaction was as follows: 4 minutes of incubation, 40 cycles of denaturation, annealing, and extension at $95^{\circ} \mathrm{C}$, $60^{\circ} \mathrm{C}$, and $72^{\circ} \mathrm{C}$ respectively. Fluorescence was measured at the completion of each extension cycle. TaqMan probes for PGC-1 $\alpha$, PPAR $\delta, \operatorname{PPAR} \alpha$ and HPRT were purchased from Applied 
Biosystems (Life Technologies). SYBR Green primers were designed using Primer-BLAST through PubMed. Primers were designed to produce amplicons between 70 and 150 bp, melting temperatures at $60^{\circ} \mathrm{C}$, and forced to span exon-exon junctions. Primer pairs with predicted nonspecific binding were eliminated from consideration. Primers were tested on agarose gel and imaged using ethidium bromide under ultraviolet light to confirm existence of a single signal band at desired amplicon length. Images were acquired utilizing a customized gel documentation apparatus with a Nikon Coolpix L820 digital camera. Targets were quantified using $2^{-\Delta \Delta C t}$ methods as previously described (Greene et al. 2014). Forward and reverse primer pairs utilized include: HMGCR: Forward: ACGTGGTGTGTCTATTCGCC, Reverse: CAAGCTCCCATCACCAAGGA; $S c d 1$ : Forward: GCCTCTTCGGGATTTTCTACTACA, Reverse: GCAGCCGTGCCTTGTAAGTT; Tnf $\alpha$ : Forward: TAGCCCACGTCGTAGCAAAC, Reverse: ACAAGGTACAACCCATCGGC; Srebpl: Forward:

GGGCCTTGATGGTCTCAGTT, Reverse: ATCCTTCGCCTATGCTGGTG. Primers for Ppar $\gamma$, mtif2, mtif3, Tufm, Taco1, Mfn1, Mfn2, Drp1, Opa1, Mff, Fis1, Beclin (also known as Atg6), Bnip3, and Atg7 were used as previously reported (Greene et al. 2015). Fold change of mRNA content was normalized to Hprt $\mathrm{Ct}$ values and then expressed as relative fold change from $\mathrm{NC}$ SED; Hprt Ct was not different between experimental groups.

\section{Isolation of Protein and Immunoblotting}

Isolation of protein and immunoblotting were performed as previously described in skeletal muscles (Greene et al. 2015). Approximately $45 \mathrm{mg}$ of liver was homogenized in glass dounce type homogenizers in $0.30 \mathrm{ml}$ of complete protein loading buffer $(50 \mathrm{mM}$ Tris $\cdot \mathrm{HCl}, \mathrm{pH}$ 6.8, $1 \%$ sodium dodecyl sulfate (SDS), 10\% glycerol, $20 \mathrm{mM}$ dithiothreitol, $127 \mathrm{mM} 2-$ 
mercaptoethanol, and $0.01 \%$ bromophenol blue supplemented with protease inhibitors (Roche) and phosphatase inhibitors (Sigma-Aldrich, St. Louis, MO)) according to Alliance for Cellular Signaling protocols and as described elsewhere (Greene et al. 2015). After homogenization, samples were transferred to sterile $1.5 \mathrm{~mL}$ microcentrifuge tubes, heated for 5 minutes at $95^{\circ} \mathrm{C}$ to denature protein and then centrifuged for 5 minutes at 13,000 rpm. Protein concentrations were determined using a commercially available RC/DC assay kit following manufacturer instructions (Bio-Rad, Hercules, CA). $40 \mu \mathrm{g}$ of liver protein was then loaded into 6-15\% SDSpolyacrylamide gels, depending on size of protein of interest, and transferred onto PVDF membranes (Thermo Scientific, Rockford, IL, USA). Membranes were blocked for an hour in $5 \%$ milk solution in $0.5 \%$ TBST, and then incubated in primary antibody blocking solution at $4^{\circ} \mathrm{C}$. After $12-48$ hour primary incubation, membranes were washed in TBS and incubated with appropriate secondary antibody solutions (Li-Cor Biosciences, Lincoln, NE). Membranes were imaged using a FlourChem M (Protein Simple, San Jose, CA) and protein content was normalized to Ponceau S. Primary antibodies included: COX-IV (Cell Signaling Technology, Danvers, MA, USA 4844), Cytochrome C (Cell Signaling 4272), MFN2 (Santa Cruz Biotechnology, Dallas, TX, USA sc-50331), DRP1 (Cell Signaling 14647), p62/SQSTM1 (Sigma Aldrich p0067, St. Louis, MO), LC3A/B (Cell Signaling 4108), and BNIP3 (Cell Signaling 3769). For all targets there was an n of 8-10 per group. Total LC3 content was taken as the sum density of the LC3-I and LC3-II bands.

\section{Histological Analysis}

Sections were cut at $10 \mu \mathrm{m}$ using a Leica CM1859 clinical cryostat (Leica Biosystems, Buffalo Grove, IL) and stained with Hematoxylin \& Eosin (H \& E). Sections were analyzed by 
certified hepatic pathologist FRCC and scored on a histology grading system of 0-4 as previously validated (Gibson-Corley et al. 2013), with a score of zero having no visible lesions or hydropic changes and a score of 4 indicating the formation of lipid cavities, inflammation and vacuoles in the hepatocytes The pathologist was blinded to all groups while scoring liver sections.

\section{Statistical Analysis}

The independent factors were diet (NC vs. WD) and PA (SED vs. VWR). For tissue weights, mRNA and protein contents, a diet by PA (2X2) ANOVA was employed as the global analysis for each dependent variable of interest. The comparison-wise error rate, $\alpha$, was set at 0.05 for all statistical tests. When significant $F$-ratios were found, a Tukey-Kramer post hoc analysis was used to distinguish differences among means, and data expressed as mean \pm SEM. For histology as data are ordinal in nature, scores were analyzed by Kruskal-Wallis nonparametric test, data is expressed as median ranks \pm SEM. Data were analyzed using the Statistical Analysis System (SAS, version 9.3, Cary, NC).

\section{Results}

Phenotypic data of animals demonstrated an obese phenotype

We have previously reported phenotypic data regarding this cohort of animals illustrating glucose intolerance (a surrogate measure of impaired glucose homeostasis), exercise intolerance, and $36 \%$ and $390 \%$ greater total body and epididymal fat weight, respectively, in WD fed animals compared to $\mathrm{NC}$ which corresponded to $\sim 9 \mathrm{~g}$ greater body weight in WD groups (Greene et al. 2015). VWR partially ameliorated glucose and exercise intolerance with no change in body and epididymal fat weights (Greene et al. 2015). VWR animals ran 
approximately 500-700 meters/day with no difference between diets (Greene et al. 2015). WD animals consumed approximately $300 \mathrm{kcal}$ more than NC animals across the total 8 wks of the intervention (Greene et al. 2015).

Western Diet-induced obesity resulted in greater liver weight with little change in hepatic markers of inflammation.

To better understand the phenotypes provided by our WD and VWR regimens, in addition to previously reported muscle weights we further assessed wet weights of liver, brown fat, heart and brain. Tissue weights were normalized to tibia length as an estimate of body size independent of weight, tibia weights did not differ among groups. WD groups exhibited greater liver and brown fat weight relative to tibia length compared to NC groups (30\% and 35\% respectively, $\mathrm{p}<0.05$; Figure 1A). No difference was seen in weights of heart or brain between experimental groups ( $p>0.05$; Figure 1A). Raw liver weights in WD-induced obese animals were approximately $1.4 \pm 0.2$ grams compared to $1.2 \pm 0.1$ grams for $\mathrm{NC}$-fed animals $(\mathrm{p}<0.05)$. No significant impact of VWR was seen in either feeding group on either raw or relative liver weight.

Histological examination of $\mathrm{H} \& \mathrm{E}$ staining by a certified pathologist (FRCC) demonstrated a median value of $0.0 \pm 0.22$ and $0.0 \pm 0.24$ for NC-SED and NC-VWR respectively (Figure 1B). WD-SED had a median value of $2.00 \pm 0.16$, with greater lipid accumulation as noted by the arrows, and was statistically significant compared to NC-SED and NC-VWR ( $<<0.05$, Figure 1B \& 1C). Conversely, WD-VWR had a median value of $1.0 \pm .44$ and was not statistically significant different from any other groups (Figure 1B \& 1C). 
Interestingly, the pathologist did not note any inflammation in the examined sections within any of the groups (Figure 1C). Lipid vacuoles in the $\mathrm{H} \& \mathrm{E}$ staining are denoted by black arrows. Of note, Oil Red O staining demonstrated similar effects with formation of lipid vacuoles observed in WD-SED (data not shown).

Stearoyl-CoA desaturase-1 (Scd1) mRNA content was 2-fold greater in WD animals compared to NC animals (p<0.05, Figure 1D). HMG-CoA-Reductase $(H M G C R)$ mRNA content was approximately 5-fold greater in NC animals compared to WD animals, which appears to have been largely driven by NC-VWR animals, though no significant interaction of diet and activity was noted ( $\mathrm{p}<0.05$, Figure 1D). mRNA content of Sterol regulatory element-binding protein (Srebp1) was 1.5 fold greater in VWR animals compared to SED animals with no significant effect of diet ( $\mathrm{p}<0.05$, Figure 1D). Corroborating the histology data, there was no significant difference in mRNA content of inflammatory markers $\operatorname{Tn} f \alpha$ between groups, although we did note higher Il6 in in NC-VWR compared to WD-VWR ( $p<0.05$, Figure 1E). Furthermore, consistent with hepatic Tnf $\alpha$ and Il6 mRNA no inflammation was noted by pathologist in histological analysis of these tissues (Gibson-Corley et al. 2013) (Figure 1C).

Markers of hepatic mitochondrial content and biogenesis are altered with Western Diet-induced obesity

CoxIV, a typical measure of mitochondrial content, had a 2-fold increase in mRNA content in NC-VWR animals compared to NC-SED animals ( $\mathrm{p}<0.05$, Figure 2A). Further, NCVWR was also approximately 2 -fold greater than WD-SED animals ( $<<0.05$, Figure $2 A)$. COXIV protein content was approximately $40 \%$ lower in WD-fed animals compared to controls with no significant effect of VWR ( $\mathrm{p}<0.05$, Figure 2B \& 2D). Cytochrome C protein content, another 
marker of mitochondrial content, was approximately 50\% less in NC-VWR, WD-SED, and WDVWR compared to NC-SED ( $p<0.05$, Figure $2 \mathrm{C} \& 2 \mathrm{D})$.

Pgcl $\alpha$ mRNA content was approximately 50\% lower in WD fed animals than that observed in NC fed animals ( $\mathrm{p}<0.05$, Figure 3A). Furthermore, Ppar $\delta$ mRNA content was approximately $60 \%$ lower in the WD fed animals compared to NC animals $(\mathrm{p}<0.05$, Figure $3 \mathrm{~B})$. Ppara mRNA content was not different in any groups (Figure 3C). Ppary mRNA content was not different between any groups (Figure 3C). Mitochondrial mRNA translation gene content of mtIF2, mtIF3, TUFM, and translational activator of mitochondrially-encoded cytochrome c oxidase I (Taco1) were not affected by diet or PA interventions ( $p>0.05$ for all, Figure 3D).

Mitochondrial fusion and fission were decreased with Western diet-induced obesity.

There were no differences in $M f n 1 \mathrm{mRNA}$ content between groups ( $\mathrm{p}>0.05$, Figure 4A). Mfn2 mRNA was approximately $40 \%$ greater in VWR animals compared to SED animals ( $\mathrm{p}<0.05$, Figure 4B). Though not significant $M f n 2 \mathrm{mRNA}$ content appeared to be lower in WD fed animals ( $\mathrm{p}=0.08$, Figure 4B); however, MFN2 protein content was $50 \%$ lower in WD animals compared to NC animals ( $\mathrm{p}<0.05$, Figure 4D). Opal mRNA content was lower in WD animals by approximately $60 \%$ ( $\mathrm{p}<0.05$, Figure 4C). With regard to fission, Mff and Drpl mRNA content were $60 \%$ and $75 \%$ lower respectively in WD compared to NC animals $(\mathrm{p}<0.05$, Figures 4F \& 4G). Furthermore, DRP1 protein content was approximately $30 \%$ lower in WD fed animals compared to NC fed counterparts (p<0.05, Figure 4I). Fis 1 mRNA was not significantly different between groups ( $\mathrm{p}>0.05$, Figure $4 \mathrm{H}$ ). 
Autophagy machinery was lower in WD, but autophagosome formation and clearance was increased in VWR animals regardless of diet.

A main effect for Beclin and Atg7 mRNA content to be lower in WD animals compared to $\mathrm{NC}$ approached significance $(\mathrm{p}=0.07$, Figure $5 \mathrm{~A} \& 5 \mathrm{~B})$. There was also a mean decrease in total LC3 content in WD fed animals compared to NC fed ( $\mathrm{p}<0.05$, Figure 5C).

. In the current study, LC3-II was also about 50\% higher in VWR compared to SED, and approximately $40 \%$ lower in WD compared to NC animals ( $p<0.05$, Figure 5D). The LC3II/I ratio was $50 \%$ greater in VWR animals compared to SED regardless of diet ( $<<0.05$, Figure 5E). Furthermore, p62 protein content was approximately $40 \%$ lower in VWR animals regardless of diet, suggesting increased autophagy ( $\mathrm{p}<0.05$, Figure 5F). Regarding regulation of mitophagy, Bnip3 mRNA appeared lower in WD animals though this effect did not reach statistical significance, $(\mathrm{p}=0.06$, Figure $5 \mathrm{G})$. BNIP3 protein content was approximately $30 \%$ lower in WD fed animals compared to $\mathrm{NC}$ animals $(\mathrm{p}<0.05$, Figure $5 \mathrm{H})$. 


\section{Discussion}

To our knowledge, this is the first investigation to demonstrate dysregulation of mitochondrial quality control processes across biogenesis, dynamics and autophagy in liver following Western Diet-induced obesity. Of note, these effects occurred despite only moderate hepatic weight gain, no signs of hepatic inflammation either by RNA or pathology, and subclinical pathology scores. This suggests that obesity-induced mitochondrial dysregulation occurs prior to the onset of NAFLD. Furthermore, pathology scores suggest potential protection against hepatic degeneration by the inclusion of concurrent moderate voluntary physical activity. Therefore, we suggest that PA-induced promotion of autophagosome formation and clearance as a result of VWR may serve to protect against the degeneration of the liver in diet-induced obesity.

Obesity is a primary risk factor for NAFLD and eventual NASH. Our WD was sufficient to increase body weight and epididymal fat as previously reported in these animals (Greene et al. 2015) as well as increase liver mass, which appears to be smaller than prior studies with more dramatic liver weights (Chen et al. 2014). Previous studies have noted increased levels of inflammatory markers (Joong Park et al. 2010) with the onset of NAFLD, whereas we found no increase in inflammation as measured by Tnf $\alpha$ and IL6, which was further confirmed by pathology. Furthermore, pathology demonstrated WD-SED animals reached a median score of 2 which was greater than both NC fed groups and is considered moderate on the scale used in which slides were rated 0-4, all of which are sub-clinical (Burt et al. 2015). In contrast, in WDVWR animals were not statistically different from either WD-SED or NC groups, suggesting at least some protection against hepatic degeneration by moderate physical activity. Taken together, these data suggest that we induced an obese phenotype, but had not yet reached a NAFLD 
phenotype in these animals, providing a unique insight into the early stages in the development of the disease. We recognize our animals exhibit low wheel running activity for this strain, while we cannot be certain as to why our animals were less active than previously observed, it is significant that even with the modest physical activity there were still some measurable benefits for hepatic degeneration.

Considering these effects and mitochondrial dysfunction has been observed in NAFLD (Rector et al. 2010) we next turned our attention to the assessment of mitochondrial quality control regulators. Our data demonstrates reduced mitochondrial content as a result of WD as measured by COX-IV and Cytochrome C. Based on this finding and previous research it is possible to postulate that reduced mitochondrial content, in addition to constant lipid overload, may lead to excess mitochondrial reactive oxygen species (ROS) emission (Gusdon et al. 2014) and consequently the development of NAFLD. Unfortunately, at this time we are unable to speculate what mechanism may have allowed reductions in Cytochrome C in NC VWR animals. While not measured directly, the repression in mitochondrial content and quality control markers in WD animals is suggestive of early impairments in biogenesis, fusion and fission in these animals which precede development of NAFLD. Admittedly, a limitation to the current study is that we were unable to directly measure mitochondrial biogenesis, dynamics, function and degeneration. However, prior studies have demonstrated direct mitochondrial dysfunction (Rector et al. 2010; Fletcher et al. 2014) in hepatic lipid overload. Therefore, our goal was to identify signaling disruptions in mitochondrial quality control that may be critical to these occurrences and provide insight to viable therapeutic targets in hepatic degeneration.

Significant past research has implicated dysfunction in autophagy machinery, and autophagosome formation and clearance as a contributing factor in NAFLD (Mei et al. 2011; 
González-Rodríguez et al. 2014; Kwanten et al. 2014; Baena et al. 2015). We note significant repressions in total LC3 and BNIP3 contents along with suggestions of lowered mRNA of Beclin, Atg7 and Bnip3 mRNAs in WD fed animals, suggesting a repression in some aspects of autophagy machinery at this stage.

Relative to mitophagy, BNIP3 is a specific regulator of this process (Andres et al. 2015) and repression of BNIP3, as observed, likely represents diminished capacity to autophagically remove mitochondria. However, such an impact of obesity on mitophagy needs to be further explored to verify our interpretations. Previous work from our lab has suggested that mitophagy may be driven by PGC-1 $\alpha$ (Greene et al. 2015). Therefore, the reduction of mitochondrial biogenesis, mitochondrial content and mitophagy may suggest that reduced mitochondrial biogenesis precedes alterations in mitophagy which would result in reduced mitochondrial content and a concurrent decrease in mitophagy. Alternately, reduced BNIP3 protein could represent greater mitophagy as a result of degradation of the protein itself during the autophagy process. However, Bnip3 mRNA content showed a similar tendency in WD fed animals as the protein suggesting lower content is due to the gene product being repressed and hence lowered mitophagy. We should note that an apparent reduction in Bnip3 mRNA in NC-VWR animals did not reach statistical significance.

This report adds to the growing body of knowledge demonstrating both increased autophagosomal formation and resolution with PA despite lipid overload in the liver. To assess basal autophagosome formation and clearance, we performed dual immunoblots of LC3 and p62/SQSTM1 similar to prior works (Zhang et al. 2012; Lira et al. 2013; Greene et al. 2015). This methodology is recognized as a valid means to assess autophagosome formation and degradation by the "Guidelines for the use and interpretation of assays for monitoring 
autophagy" by Klionsky et al. (Klionsky et al. 2016). Briefly, LC3 is altered upon induction of autophagy via phosphatidylethanolamine (PE)-conjugatation to form what is termed the LC3-II form of LC3 and becomes attached to the membrane of the autophagosome, while LC3 presents in the unconjugated LC3-I form when autophagy is not activated. Therefore the ratio of LC3-II to LC3-I provides significant insight to rates of induction of autophagy and has been seen it prior publications to match well with the formation of LC3 puncta, indicative of autophagosomes (He et. al, 2012) By contrast, p62 is a cargo protein which functions in bringing aggregate proteins and damaged organelles into the forming autophagosome, as a result upon autophagy resolution p62 itself is degraded and therefore the reduction in p62 content is an indicator for resolution of the autophagy process (Klionsky et. al, 2016). As such, the combination of these immunoblots provides insight to autophagy activation and resolution, which likely suggests enhanced removal of damaged products and thereby a healthier intracellular environment. Our data of increased LC3II/I ratio and reduced p62 content both in VWR animals suggest an increase in autophagosome formation as well as an increase in autophagosome clearance. Using the same combination immunoblot methodology, Lira et al. (Lira et al. 2013) demonstrated that exercise training enhances basal autophagy flux in skeletal muscles and that autophagy is necessary for exercise-induced skeletal muscle adaptations. Furthermore, recent work by Santos-Alves et al. has found similar alterations in LC3II content as well as LC3II/I ratios in the livers of both treadmill and wheel trained rats, though they noted either no difference or an increase in p62 protein content (Santos-Alves et al. 2015). This discrepancy may be due to differences between rodent models (rats v. mice) or may be attributable to differences in animal sacrifice protocols (48 hrs v. 24 hrs post-exercise) (Santos-Alves et al. 2015). These postulates are beyond the scope of this paper, but warrant further investigation. We have in the current study added to their 
findings in demonstrating that the increase in LC3II/I ratio and the newly observed reduction in p62 by VWR occur in both NC and WD fed animals suggesting this impact of exercise/physical activity persists despite the obese phenotype and may serve as a protective mechanism in the hepatocyte. We should acknowledge that in the current study we cannot be fully certain that observed changes in p62 protein are fully attributed to autophagosome clearance and not affected by changes in p62 expression. As others have noted similar findings with exercise and alterations in p62 closely follow those in LC3 assessment of autophagosome formation we have interpreted these data as enhanced clearance, an interpretation that warrants further validation. Future studies should seek to corroborate our findings using additional measures of autophagy including the use of fluorescently tagged LC3 to assess puncta (autophagosome) formation and inclusion of lysosomal inhibitors allowing the tracking of autophagosome formation over a period of time. We acknowledge that in the current study by our point measurement we are unable to assess autophagic activity over time and thereby interpretations to flux over time are limited. We chose in the current study to avoid these measures to reduce any risk of unintentionally perturbing the system to maintain a basal condition at time of assessment as best as possible.

Furthermore, it is notable that autophagy is the only cellular process dramatically affected by physical activity in the current study. It is also of interest that promotion of autophagy occurred regardless of the obese phenotype. As genetic models of autophagy deficiency induce liver disease (Glick et al. 2012), and autophagy was the only cellular process dramatically affected by PA we speculate that promotion of autophagy by PA, which appeared to ameliorate hepatic histopathological disruptions, may promote protections against hepatic degeneration despite lipid overload. Future studies should determine if promotion of hepatic autophagy can indeed protect or rescue liver maladaptations during lipid overload as well as corroborate our 
findings using additional methodologies. Our combined evidence suggests that at this stage of disease progression general autophagy machinery is repressed, while specific mitophagy is impaired.

In summary, our data suggest that WD-induced obesity represses some mitochondrial quality control mechanisms with only 8 wks of moderate lipid overload though we do recognize the measured markers of our study only show a small portion of these processes and not the entire picture. These mitochondrial impairments may precede other detriments associated with NAFLD and NASH such as inflammation and increased fatty acid synthesis. We are also the first to provide evidence of enhanced autophagosome formation and resolution in the liver following habitual PA, regardless of lipid overload. This may provide a novel mechanism by which PA protects against hepatic degeneration during pre-steatotic and NAFLD conditions. 
Conflicts of Interest: The authors report no conflicts of interest associated with this manuscript.

Acknowledgements: This work was funded by the University of Arkansas. The authors would like to thank the dedicated faculty, staff, and students of the University of Arkansas Human Performance Laboratory, Dr. Sami Dridi and Mr. Aaron Caldwell for technical assistance with this project. We would also like to thank Dr. Joshua Wooten of Southern Illinois University Edwardsville for his critical review of our work. 


\section{References}

Almeda-Valdes, P., Aguilar-Olivos, N., Uribe, M., and Mendez-Sanchez, N. 2014. Common Features of the Metabolic Syndrome and Nonalcoholic Fatty Liver Disease. Rev. Recent. Clin. Trials. 9(3): 148-58. doi: 10.2174/1574887109666141216103908. PMID: 25514910.

Andres, A.M., Stotland, A., Queliconi, B.B., and Gottlieb, R.A. 2015. A time to reap, a time to sow: mitophagy and biogenesis in cardiac pathophysiology. J. Mol. Cell Cardiol. 78: 62-72. doi: 10.1016/j.yjmcc.2014.10.003. PMID:25444712.

Baena, M., Sangüesa, G., Hutter, N., Sánchez, R.M., Roglans, N., Laguna, J.C. et al. 2015. Fructose supplementation impairs rat liver autophagy through mTORC activation without inducing endoplasmic reticulum stress. Biochim. Biophys. Acta. 1851(2): 107-16. doi: 10.1016/j.bbalip.2014. PMID: 25463011.

Burt, A.D., Lackner, C., and Tiniakos, D.G. 2015. Diagnosis and Assessment of NAFLD: Definitions and Histopathological Classification. Semin. Liver Dis. 35(3): 207-20. doi: 10.1055/s-0035-1562942. PMID: 26378639.

Cai, N., Zhao, X., Jing, Y., Sun, K., Jiao, S., Chen, X. et al. 2014. Autophagy protects against palmitate-induced apoptosis in hepatocytes. Cell. Biosci. 4: 28. doi: 10.1186/2045-3701-4-28. PMID:24904743.

Centers for Disease Control. 2007. Behavioral Risk Factor Surveillance System Survey Data. Atlanta, Georgia: U.S. Department of Health and Human Services, Centers for Disease Control and Prevention.

Chen, H.L., Tung, Y.T., Tsai, C.L., Lai, C.W., Lai, Z.L., Tsai, H.C., et al. 2014. Kefir improves fatty liver syndrome by inhibiting the lipogenesis pathway in leptin-deficient ob/ob knockout mice. 38(9): 1172-9. doi: 10.1038/ijo.2013.236. PMID:24335764. 
Fletcher, J.A., Meers, G.M., Linden, M.A., Kearney, M.L., Morris, E.M., Thyfault, J.P. et al. 2014. Impact of various exercise modalities on hepatic mitochondrial function. Med. Sci. Sports Exerc. 46(6): 1089-97. doi: 10.1249/MSS.0000000000000223. PMID:24263979.

Funai, K., Song, H., Yin, L., Lodhi, I.J., Wei, X., Yoshino, J., Coleman, T. et al. 2013. Muscle lipogenesis balances insulin sensitivity and strength through calcium signaling. J. Clin. Invest. 123(3): 1229-40. doi: 10.1172/JCI65726. PMID:23376793.

Galloway, C.A., Lee, H., Brookes, P.S., and Yoon, Y. 2014. Decreasing mitochondrial fission alleviates hepatic steatosis in a murine model of nonalcoholic fatty liver disease. Am. J. Physiol. Gastrointest. Liver Physiol. 307(6): G632-41. doi: 10.1152/ajpgi.00182. PMID:25080922.

Gibson-Corley, K.N., Olivier, A.K., and Meyerholz, D.K. 2013. Principles for valid histopathologic scoring in research. Vet. Pathol. 50(6): 1007-15. doi: 10.1177/0300985813485099. PMID:23558974.

Glick, D., Zhang, W., Beaton, M., Marsboom, G., Gruber, M., Simon, M.C. et al. 2012. BNip3 regulates mitochondrial function and lipid metabolism in the liver. Mol. Cell. Biol. 32(13): 257084. doi: 10.1128/MCB.00167-12. PMID:22547685.

Goncalves, I.O. et al. 2016. Exercise mitigates mitochondrial permeability transition pore and quality control mechanisms alterations in nonalcoholic steatohepatitis. Appl Physiol Nutr Metab. 41(3):298-306. doi: 10.1139/apnm-2015-0470. PMID:26905378.

González-Rodríguez, A., Mayoral, R., Agra, N., Valdecantos, M.P., Pardo, V., MiquilenaColina, M.E. et al. 2014. Impaired autophagic flux is associated with increased endoplasmic reticulum stress during the development of NAFLD. Cell Death. Dis. 5: e1179. doi: 10.1038/cddis. PMID:24743734.

Greene, N.P., Lee, D.E., Brown, J.L., Rosa, M.E., Brown, L.A., Perry, R.A.J. et al. 2015. Mitochondrial quality control, driven by PGC-1 $\alpha$, is dysregulated by Western Diet-induced 
obesity and partially restored by moderate physical activity in mice. Physiol. Reports. 3: e12470. doi: 10.14814/phy2.12470. PMID:26177961.

Greene N.P., Washington T.A., Lee D.E., Brown L.A., Papineau A.M., Shimkus K.L. et al. 2014. Impaired exercise-induced mitochondrial biogenesis in the obese Zucker rat, despite PGC$1 \alpha$ induction, is due to compromised mitochondrial translation elongation. Am. J. Physiol. Endocrinol. Metab. 2014:E503-11. doi: 10.1152/ajpendo.00671. PMID:24398401. Guo, R., Liong, E.C., So, K.F., Fung, M.L., and Tipoe, G.L. 2015. Beneficial mechanisms of aerobic exercise on hepatic lipid metabolism in non-alcoholic fatty liver disease. Hepatobiliary Pancreat. Dis. Int. 14(2): 139-44. PMID:25865685.

Gusdon, A.M., Song, K.X., and Qu, S. 2014. Nonalcoholic Fatty liver disease: pathogenesis and therapeutics from a mitochondria-centric perspective. Oxid. Med. Cell Longev. 2014: 637027. doi: 10.1155/2014/637027. PMID:25371775.

He, Y., Zhao, X., Gao, J., Fan, L., Yang, G., Cho, W.C. et al.. 2012. Quantum dots-based immunofluorescent imaging of stromal fibroblasts Caveolin-1 and light chain 3B expression and identification of their clinical significance in human gastric cancer. Int J Mol. Sci. 13(11): 13764-80. doi: 10.3390/ijms131113764. PMID: 23203033.

Joong Park, E., Hee Lee, J., Yu, G.-Y., He, G., Raza Ali, S., Holzer, R. et al. 2010. Dietary and Genetic Obesity Promote Liver Inflammation and Tumorigenesis by Enhancing IL-6 and TNF Expression. Cell. 2010:197-208. doi: 10.1016/j.cell.2009.12.052. PMID:20141834.

Klionsky, D.J., Abdelmohsen, K., Abe, A., Abedin, M.J., Abeliovich, H., Acevedo Arozena, A. et al. 2016. Guidelines for the use and interpretation of assays for monitoring autophagy (3rd edition). Autophagy. 12(1): 1-222. doi: 10.1080/15548627.2015.1100356. PMID:26799652. 
Kwanten, W.J., Martinet, W., Michielsen, P.P., and Francque, S.M. 2014. Role of autophagy in the pathophysiology of nonalcoholic fatty liver disease: a controversial issue. World J.

Gastroenterol. 20(23): 7325-38. doi: 10.3748/wjg.v20.i23.7325. PMID:24966603.

Lavallard, V.J. and Gual, P. 2014. Autophagy and non-alcoholic fatty liver disease. Biomed. Res. Int. 2014: 120179.

Lavoie, J.M. and Gauthier, M.S. 2006. Regulation of fat metabolism in the liver: link to nonalcoholic hepatic steatosis and impact of physical exercise. Cell Mol. Life Sci. 63(12): 1393-409. PMID:16649140.

Lee, D.E., Brown, J.L., Rosa, M.E., Brown, L.A., Perry, R.A., Wiggs, M.P. et al. 2015. microRNA-16 Is Downregulated During Insulin Resistance and Controls Skeletal Muscle Protein Accretion. J. Cell Biochem. 2015. doi: 10.1002/jcb.25476.

Lira, V.A., Okutsu, M., Zhang, M., Greene, N.P., Laker, R.C., Breen, D.S. et al. 2013. Autophagy is required for exercise training-induced skeletal muscle adaptation and improvement of physical performance. FASEB J. 27(10): 4184-93. doi: 10.1096/fj.13-228486.

PMID:23825228.

Mei, S., Ni, H., Manley, S., Bockus, A., Kassel, K.M., Luyendyk, J.P. et al. 2011. Differential Roles of Unsaturated and Saturated Fatty Acids on Autophagy and Apoptosis in Hepatocytes. J. Pharmacol. Exp. Ther. 2011: 487-498. doi: 10.1124/jpet.111.184341. PMID:21856859. Mizushima, N., Levine, B., Cuervo, A.M., and Klionsky, D.J. 2008. Autophagy fights disease through cellular self-digestion. Nature. 451(7182): 1069-75. doi: 10.1038/nature06639. PMID:18305538. 
Mokdad AH, F.E., Bowman BA, Dietz WH, Vinicor F, Bales VS, and Marks JS. (2003).

Prevalence of obesity, diabetes, and obesity-related health risk factors, 2001. JAMA. 2003: 76-

79. PMID:12503980.

Perfield, J.W., Ortinau, L.C., Pickering, R.T., Ruebel, M.L., Meers, G.M., and Rector, R.S. 2013. Altered hepatic lipid metabolism contributes to nonalcoholic fatty liver disease in leptin-deficient Ob/Ob mice. J. Obes. 2013: 296537. doi: 10.1155/2013/296537. PMID:23401753.

Pessayre, D. (2007). Role of mitochondria in non-alcoholic fatty liver disease. J. Gastroenterol. Hepatol. 22 Suppl 1: S20-7.PMID:17567459.

Rector, R.S., Thyfault, J.P., Uptergrove, G.M., Morris, E.M., Naples, S.P., Borengasser, S.J. et al. 2010. Mitochondrial dysfunction precedes insulin resistance and hepatic steatosis and contributes to the natural history of non-alcoholic fatty liver disease in an obese rodent model. J. Hepatol. 52(10): 727-36. doi: 10.1016/j.jhep.2009.11.030. PMID:20347174.

Rector, R.S., Uptergrove, G.M., Morris, E.M., Borengasser, S.J., Laughlin, M.H., Booth, F.W. et al. 2011. Daily exercise vs. caloric restriction for prevention of nonalcoholic fatty liver disease in the OLETF rat model. Am. J. Physiol. Gastrointest. Liver. Physiol. 300(5): G874-83. doi:10.1152/ajpgi.00510.2010. PMID:21350190.

Santos-Alves, E., Marques-Aleixo, I., Rizo-Roca, D., Torrella, J.R., Oliveira, P.J., Magalhães, J. et al. 2015. Exercise modulates liver cellular and mitochondrial proteins related to quality control signaling. Life Sci. 135(2015): 124-130. doi: 10.1016/j.lfs.2015.06.007. PMID:26135624. Wang et al. 2015. Disruption of mitochondrial fission in the liver protects mice from dietinduced obesity and metabolic deterioration. Diabetologia. 58(10):2371-80. doi:10.1007/s00125015-3704-7. PMID:26233250. 
Westermann, B. 2010. Mitochondrial fusion and fission in cell life and death. Nat. Rev. Mol. Cell Biol. 11(12): 872-84. doi: 10.1038/nrm3013. PMID:21102612.

Xu, J., Cao, K., Li, Y., Zou, X., Chen, C., Szeto, I.M., et al. 2014. Bitter gourd inhibits the development of obesity-associated fatty liver in C57BL/6 mice fed a high-fat diet. J. Nutr. 144(4): 475-83. doi: 10.3945/jn.113.187450. PMID:24523491.

Zhang, Z., Zhang, W., Jung, D.Y., Ko, H.J., Lee, Y., Friedline, R.H. et al. 2012. TRPM2 Ca2+ channel regulates energy balance and glucose metabolism. Am. J. Physiol. Endocrinol. Metab. 302(7): E807-16. doi: 10.1152/ajpendo.00239.2011. PMID:22275755. 


\section{Figure Captions:}

Figure 1. Phenotypic data for animal groups (NC-SED, NC-VWR, WD-SED, WDVWR) and fat metabolism and inflammation markers after 8 weeks of diet and 4 weeks of activity interventions. A: Tissue weights from study normalized to tibia length. Raw liver weights in WD-induced obese animals were approximately $1.4 \pm 0.2$ grams compared to $1.2 \pm$ 0.1 grams for NC-fed animals $(\mathrm{p}<0.05)$. B: Histology Ratings post interventions, where $0=$ no visible lesions, $1=$ numerous small vacuoles in cytoplasm, $2=$ one large vacuole in cytoplasm causing change in the location of nucleus, increased hepatocyte size, $3=$ formation of lipid cavities due to coalescing hepatocytes, hepatocytes greater than twice the normal size, 4=formation multiple lipid cavities in hepatocytes. C: Representative histology images. Lipid vacuoles in stain are highlighted by black arrows. D: mRNA content of $S c d 1, H M G C R$, and Srebp1. E: mRNA content of inflammation markers Tnf $\alpha$ and IL6. Fold change of mRNA content was normalized to Hprt $\mathrm{Ct}$ values and then expressed as relative fold change from NCSED; Hprt Ct was not different between experimental groups. n of 8-10 per group. Data are expressed as mean \pm SEM. ${ }^{*} \mathrm{p}<0.05$ between indicated groups.

Figure 2. mRNA and protein content of mitochondrial content markers. A: CoxIV mRNA content B: COX-IV protein content. C: Cytochrome C protein content. D: Representative blots of B and C. Fold change of mRNA content was normalized to Hprt $\mathrm{Ct}$ values and then expressed as relative fold change from NC-SED; Hprt Ct was not different between experimental groups Fold change of protein content was normalized to Ponceau $S$ expressed as relative fold change from NC-SED. $n$ of 8-10 per group. Data are expressed as mean \pm SEM. $* p<0.05$ between indicated groups. 
Figure 3. Mitochondrial Biogenesis markers. A: $P g c l \alpha$ mRNA content. B: mRNA content of

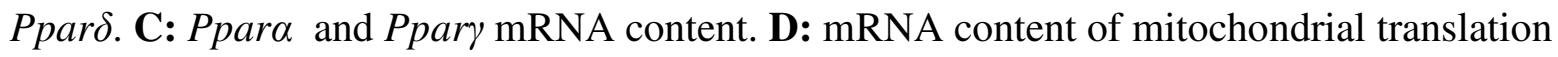
markers mtif2, mtif3, Tufm, and Tacol. Fold change of mRNA content was normalized to Hprt $\mathrm{Ct}$ values and then expressed as relative fold change from NC-SED; Hprt $\mathrm{Ct}$ was not different between experimental groups Fold change of protein content was normalized to Ponceau $S$ expressed as relative fold change from NC-SED. $\mathrm{n}$ of 8-10 per group. Data are expressed as mean \pm SEM. ${ }^{*} \mathrm{p}<0.05$ between indicated groups.

Figure 4. Mitochondrial Dynamics for the four groups divided into Fusion and Fission. A: Mfnl mRNA content. B: $M f n 2$ mRNA content. C: Opal mRNA content. D: MFN2 protein content E: Representative blot for MFN2. F: Mff mRNA content. G: Drp1 mRNA content. H: Fis1 mRNA content. I: DRP1 protein content. J: Representative blot for DRP1. Fold change of mRNA content was normalized to Hprt $\mathrm{Ct}$ values and then expressed as relative fold change from NC-SED; Hprt Ct was not different between experimental groups Fold change of protein content was normalized to Ponceau $S$ expressed as relative fold change from NC-SED. n of 8-10 per group. Data are expressed as mean \pm SEM. ${ }^{*} \mathrm{p}<0.05$ between indicated groups.

Figure 5. Autophagy machinery and markers of autophagosomal formation among groups after 8 weeks of diet and 4 weeks of PA interventions. A: Beclin mRNA content. B: Atg7 mRNA content. C: Total LC3 protein content. D: LC3II protein content. E: LC3II/I protein content ratios. F: p62 protein content. G: Bnip3 mRNA content. H: BNIP3 protein content. I: Representative western blot images. Fold change of mRNA content was normalized to Hprt $\mathrm{Ct}$ 
values and then expressed as relative fold change from NC-SED; Hprt $\mathrm{Ct}$ was not different between experimental groups Fold change of protein content was normalized to Ponceau $S$ expressed as relative fold change from NC-SED. $\mathrm{n}$ of 8-10 per group. Data are expressed as mean \pm SEM. ${ }^{*} \mathrm{p}<0.05$ between indicated groups. 
A
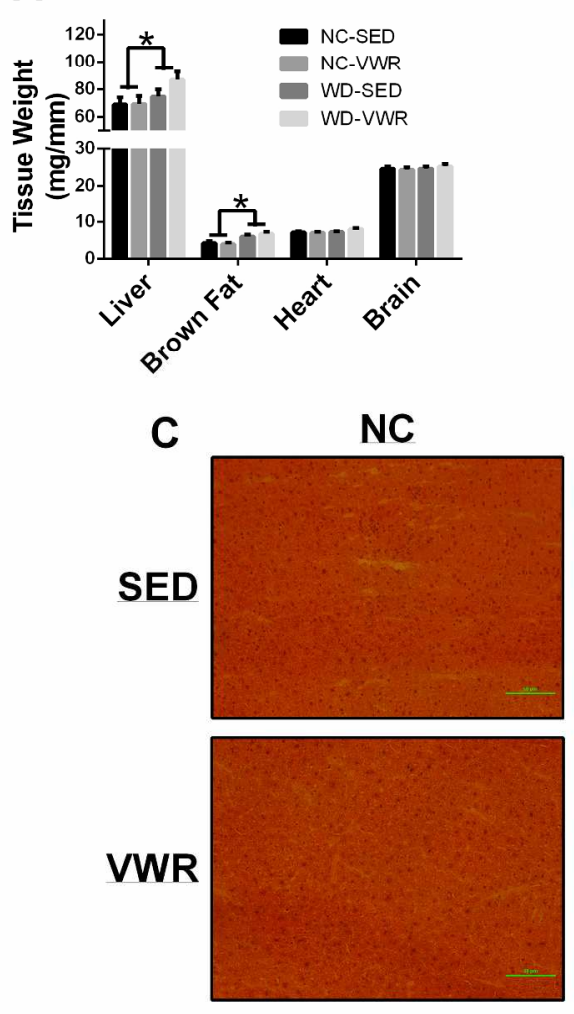

D

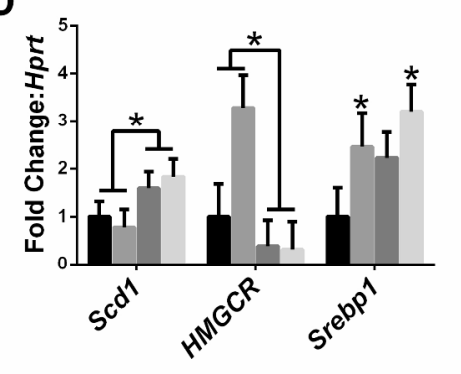

B Histology Ratings

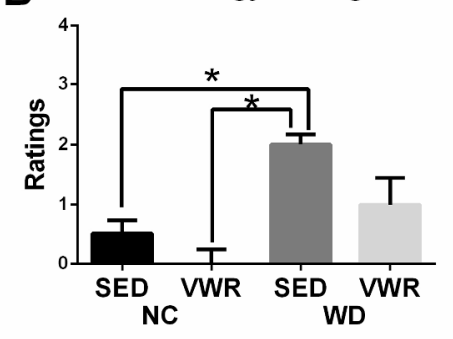

WD
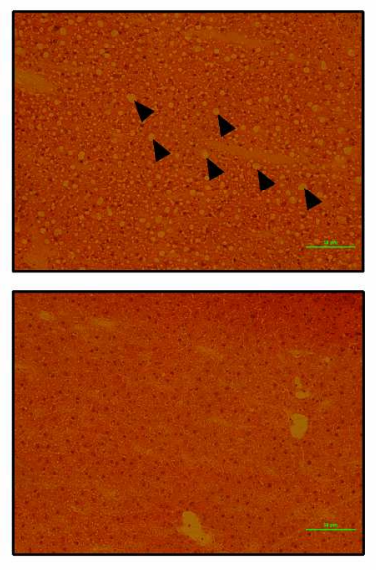

E

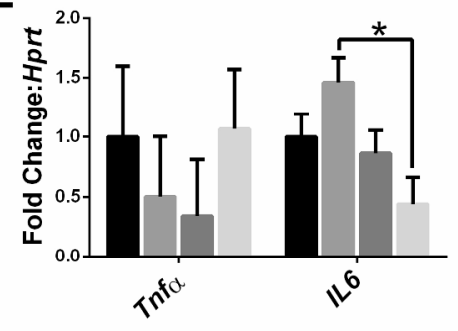

Figure 1. Phenotypic data for animal groups (NC-SED, NC-VWR, WD-SED, WD-VWR) and fat metabolism and inflammation markers after 8 weeks of diet and 4 weeks of activity interventions. A: Tissue weights from study normalized to tibia length. Raw liver weights in WD-induced obese animals were approximately $1.4+$ 0.2 grams compared to $1.2+0.1$ grams for NC-fed animals $(p<0.05)$. B: Histology Ratings post interventions, where $0=$ no visible lesions, $1=$ numerous small vacuoles in cytoplasm, $2=$ one large vacuole in cytoplasm causing change in the location of nucleus, increased hepatocyte size, $3=$ formation of lipid cavities due to coalescing hepatocytes, hepatocytes greater than twice the normal size, $4=$ formation multiple lipid cavities in hepatocytes. C: Representative histology images. Lipid vacuoles in stain are highlighted by black arrows. D: mRNA content of Scd1, HMGCR, and Srebp1. E: mRNA content of inflammation markers Tnfa and IL6. Fold change of mRNA content was normalized to Hprt Ct values and then expressed as relative fold change from NC-SED; Hprt Ct was not different between experimental groups. $\mathrm{n}$ of 8-10 per group. Data are expressed as mean + SEM. $* \mathrm{p}<0.05$ between indicated groups. 
$216 \times 282 \mathrm{~mm}(300 \times 300 \mathrm{DPI})$ 
A

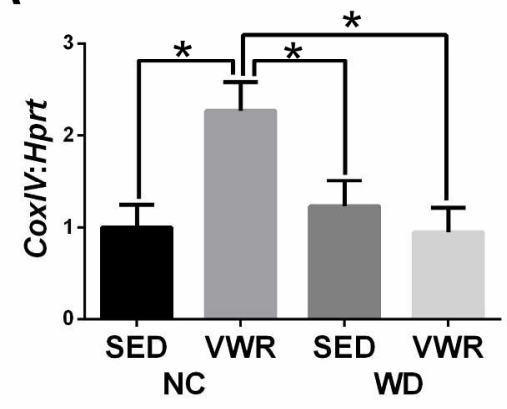

C

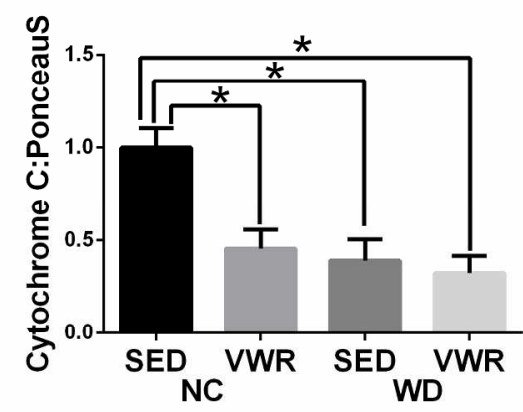

B

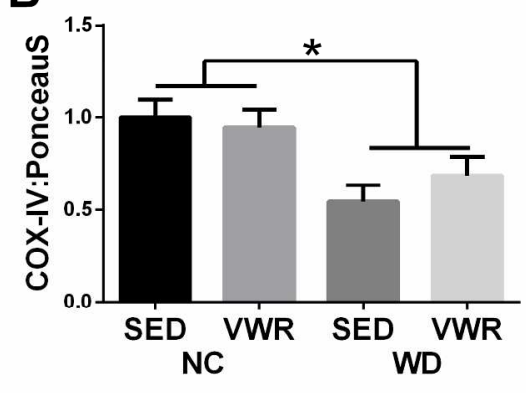

D

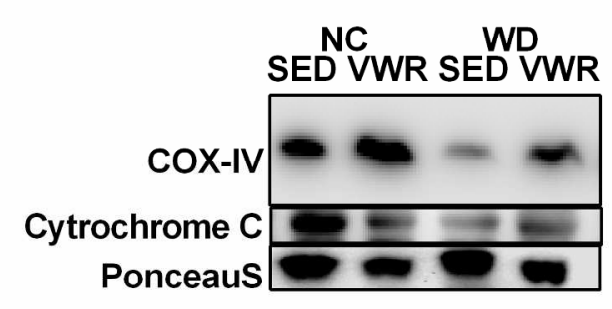

Figure 2. mRNA and protein content of mitochondrial content markers. A: CoxIV mRNA content B: COX-IV protein content. C: Cytochrome $C$ protein content. D: Representative blots of $B$ and C. Fold change of mRNA content was normalized to Hprt Ct values and then expressed as relative fold change from NC-SED; Hprt Ct was not different between experimental groups Fold change of protein content was normalized to Ponceau $\mathrm{S}$ expressed as relative fold change from NC-SED. $\mathrm{n}$ of 8-10 per group. Data are expressed as mean + SEM. ${ }^{*} p<0.05$ between indicated groups. 
A

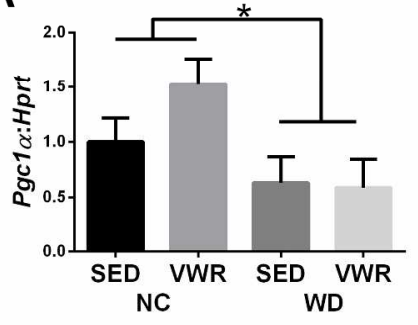

C

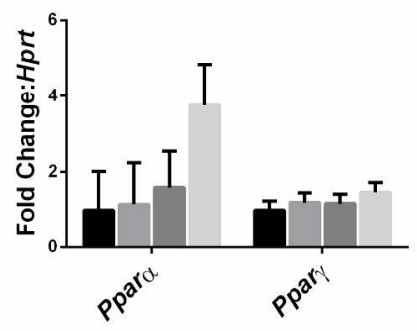

B

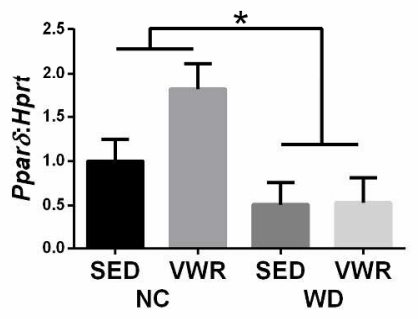

D

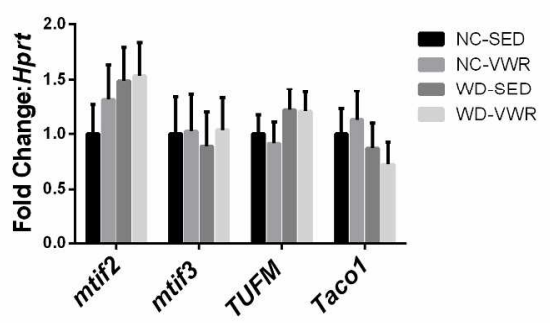

Figure 3. Mitochondrial Biogenesis markers. A: Pgc1a mRNA content. B: mRNA content of Pparס. C: Ppara and Ppary mRNA content. D: mRNA content of mitochondrial translation markers mtif2, mtif3, Tufm, and Taco1. Fold change of mRNA content was normalized to Hprt Ct values and then expressed as relative fold change from NC-SED; Hprt Ct was not different between experimental groups Fold change of protein content was normalized to Ponceau $S$ expressed as relative fold change from NC-SED. $n$ of 8-10 per group. Data are expressed as mean $+\mathrm{SEM} .{ }^{*} \mathrm{p}<0.05$ between indicated groups. 


\section{Fusion}
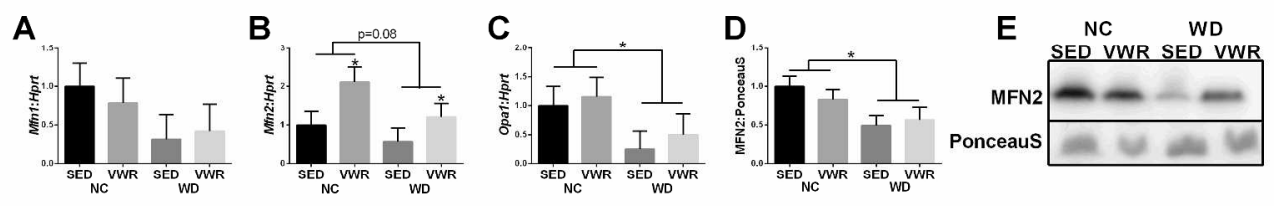

\section{Fission}
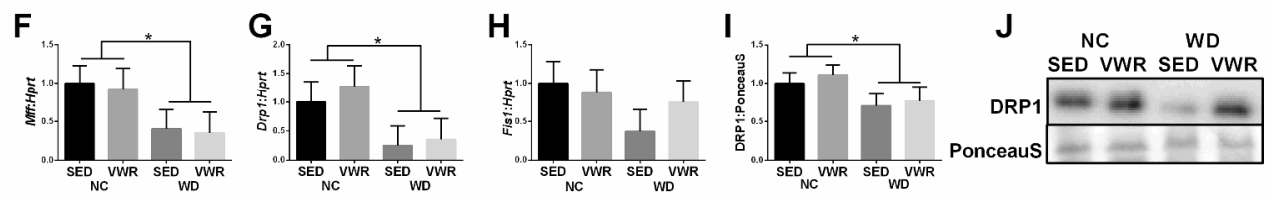

Figure 4. Mitochondrial Dynamics for the four groups divided into Fusion and Fission. A: Mfn1 mRNA content. B: Mfn2 mRNA content. C: Opa1 mRNA content. D: MFN2 protein content E: Representative blot for MFN2.

F: Mff mRNA content. G: Drp1 mRNA content. H: Fis1 mRNA content. I: DRP1 protein content. J:

Representative blot for DRP1. Fold change of mRNA content was normalized to Hprt Ct values and then expressed as relative fold change from NC-SED; Hprt Ct was not different between experimental groups Fold change of protein content was normalized to Ponceau $S$ expressed as relative fold change from NC-SED. $n$ of 8-10 per group. Data are expressed as mean + SEM. $* p<0.05$ between indicated groups. 


\section{Autophagy Machinery}
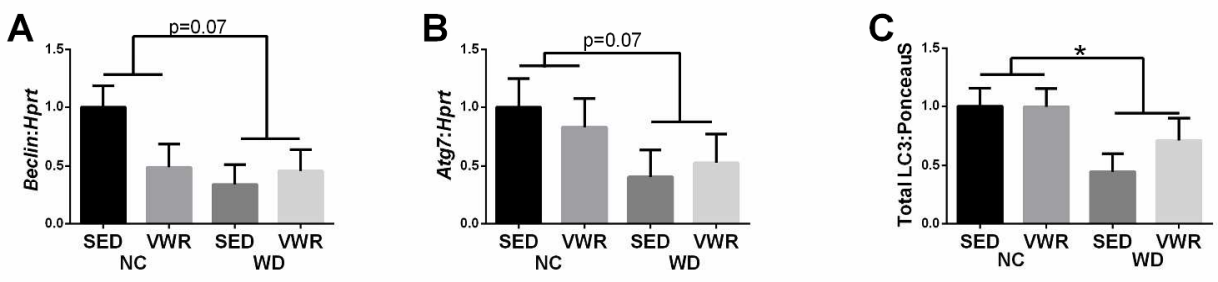

\section{Autophagosome Formation and Clearance}
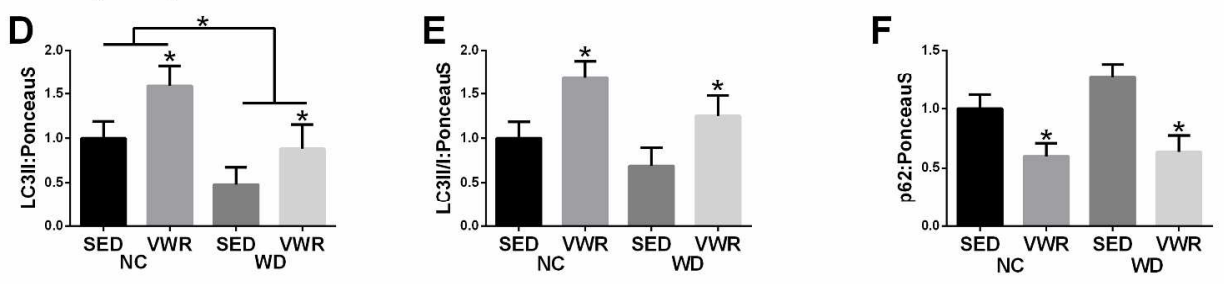

\section{Mitophagy}
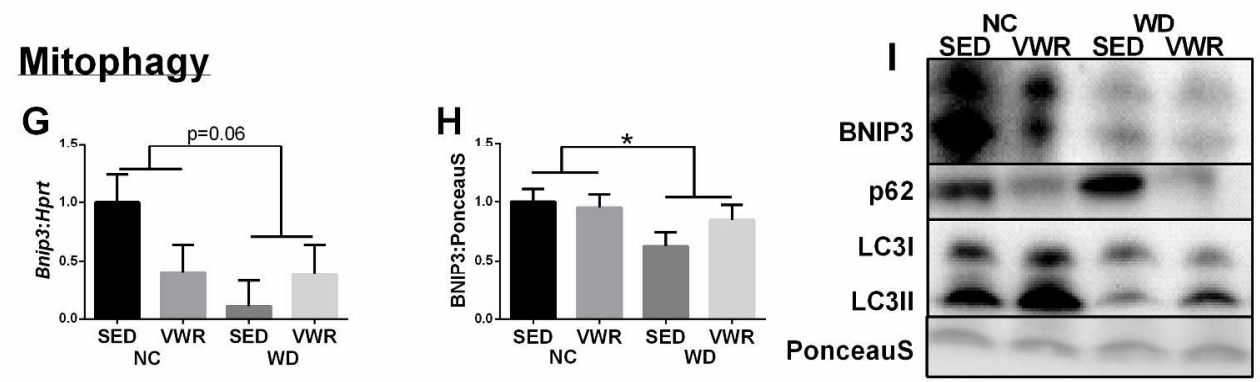

Figure 5. Autophagy machinery and markers of autophagosomal formation among groups after 8 weeks of diet and 4 weeks of PA interventions. A: Beclin mRNA content. B: Atg7 mRNA content. C: Total LC3 protein content. D: LC3II protein content. E: LC3II/I protein content ratios. F: p62 protein content. G: Bnip3 mRNA content. H: BNIP3 protein content. I: Representative western blot images. Fold change of mRNA content was normalized to Hprt Ct values and then expressed as relative fold change from NC-SED; Hprt Ct was not different between experimental groups Fold change of protein content was normalized to Ponceau $S$ expressed as relative fold change from NC-SED. $n$ of 8-10 per group. Data are expressed as mean + SEM. $* p<0.05$ between indicated groups. 